\title{
Radiosurgery for small-cell lung cancer brain metastases: a review
}

\author{
Tyler P. Robin, Chad G. Rusthoven
}

Department of Radiation Oncology, University of Colorado School of Medicine, Aurora, CO, USA

Contributions: (I) Conception and design: All authors; (II) Administrative support: None; (III) Provision of study materials or patients: None; (IV) Collection and assembly of data: None; (V) Data analysis and interpretation: None; (VI) Manuscript writing: All authors; (VII) Final approval of manuscript: All authors.

Correspondence to: Chad G. Rusthoven. Department of Radiation Oncology, University of Colorado School of Medicine, Aurora, CO, USA. Email: chad.rusthoven@cuanschutz.edu.

\begin{abstract}
First-line stereotactic radiosurgery (SRS) is now considered the preferred treatment over whole brain radiation therapy (WBRT) for limited brain metastases arising from most tumor histologies. This standard was reached following the consistent results of multiple phase III studies which demonstrated that, despite improved CNS control, the addition of WBRT to SRS does not improve overall survival (OS) and is associated with a reduction in cognitive function. Thus, it may be reasonable to consider the benchmark necessary to favor a paradigm of SRS alone over strategies incorporating WBRT as the demonstration of comparable OS in the context of decreased treatment-related side effects. However, patients with small-cell lung cancer (SCLC) brain metastases were excluded from the landmark trials that established SRS alone for limited brain metastases, largely due to concerns for short-interval CNS progression in SCLC as well the historic role of prophylactic cranial irradiation (PCI) in SCLC in the absence of known brain metastases. As a result, WBRT has remained the standard for SCLC for limited and even solitary brain lesions. With shifting SCLC care patterns including increased MRI surveillance, decreased PCI delivery, and emerging systemic agents, interest in first-line SRS for SCLC is likely to continue to increase over time. Herein we will review the emerging data for first-line SRS in the management of SCLC brain metastases and the potential for its increasing role in the setting of a greater utilization of MRI surveillance and improving systemic therapies.
\end{abstract}

Keywords: Stereotactic radiosurgery (SRS); whole brain radiation therapy (WBRT); small-cell lung cancer (SCLC); brain metastases

Submitted Mar 04, 2020. Accepted for publication Mar 16, 2020.

doi: $10.21037 /$ jtd.2020.03.90

View this article at: http://dx.doi.org/10.21037/jtd.2020.03.90

\section{Brain metastases in small-cell lung cancer (SCLC): the background}

SCLC has a high proclivity for dissemination to the brain and therefore management of brain metastases in this disease is an important topic (1). The historic standard of care for SCLC for limited and even solitary brain metastases has been whole brain radiation therapy (WBRT). Moreover, preventative WBRT, in the form of prophylactic cranial radiation $(\mathrm{PCI})$, has been traditionally offered in the absence of brain metastases due to the demonstration of improved OS with PCI in landmark studies $(2,3)$. However, WBRT has consistently been associated with neurocognitive toxicities and, as a result, in other histologies WBRT has largely been replaced by a strategy of upfront stereotactic radiosurgery (SRS) for limited brain metastases. As systemic therapies for SCLC are improving (4-6) and the contemporary OS advantage of PCI has been challenged by strategies involving brain MRI surveillance and early salvage therapy for brain metastases $(7,8)$, interest in the potential 
role of SRS in carefully selected SCLC patients is likely to grow (9).

\section{The evolution of SRS for brain metastases from other histologies}

Multiple prospective randomized trials have compared a strategy of SRS alone to SRS plus WBRT and have demonstrating that, while WBRT offers consistent improvement in CNS control, WBRT does not offer an improvement in overall survival (OS) over SRS alone (10-14). What's more, the superior CNS control achieved with WBRT appears to come at the cost of inferior cognitive performance and patient-reported QOL. In one of the first landmark trials to compare SRS to SRS plus WBRT, Aoyama et al. enrolled 132 patients in a multicenter Japanese study comparing SRS to SRS and WBRT for 1-4 brain metastases and demonstrated an improvement in CNS control but no significant differences in OS with the addition of WBRT (10). The EORTC later completed a similar study comparing WBRT to observation after local therapy with either SRS or surgical resection and similarly reported improvement in CNS progression with the addition of WBRT, but no difference in OS (14). In this study receipt of WBRT was also associated with a decrement in some areas of health-related quality-of-life (HRQOL) (15). Chang et al. conducted a single-institution phase III study of SRS alone versus SRS and WBRT for patients with 1-3 brain metastases with cognitive function as the primary endpoint. The study was stopped early by the data safety monitoring committee after a decline in cognitive function was observed in the WBRT arm at 4-month (13). Brown et al. then conducted two studies through the NCCTG that also assessed cognitive function in patients receiving WBRT and/or SRS. In the first study patients with 1-3 intact brain metastases were randomized to SRS alone or SRS and WBRT and in the subsequent study, patients with resected brain metastases were randomized to postoperative SRS to the surgical cavity or WBRT $(11,12)$. In both studies WBRT was associated with cognitive decline, worse quality-of-life, and again there were no significant differences in OS. Importantly, cognitive decline was also assessed at a later time point in long-term survivors and a persistent decrement to cognitive function with WBRT was observed (11).

In response to the consistent results of these randomized trials demonstrating improved CNS control, inferior cognitive function and QOL, and no significant differences in OS with the addition of WBRT, SRS became the guideline-endorsed standard for limited brain metastases arising from most tumor histologies (16). However, patients with SCLC were excluded from these practice-changing trials and, as a result, WBRT has remained the standard of care for SCLC brain metastases. However, in response to emerging retrospective data discussed below, the contemporary National Comprehensive Cancer Network (NCCN) SCLC treatment guidelines now acknowledge SRS as a potential treatment option for carefully selected SCLC patients with a small number of brain metastases (4).

\section{Data supporting first-line SRS for small-cell lung cancer brain metastases}

Table 1 highlights the pertinent retrospective studies including SCLC patients with brain metastases treated with first-line SRS without prior WBRT or PCI. In one of the earliest studies of first-line SRS for SCLS brain metastases, Serizawa et al. compared the results of SRS for 34 patients with SCLC with 211 patients with non-small-cell lung cancer (NSCLC) (where upfront SRS is a well established treatment strategy) and observed no significant differences in local control, OS, or neurologic survival between SCLC and NSCLC after SRS (17). Wegner et al. reviewed the outcomes for 44 patients treated with SRS for SCLC brain metastases; eight of 44 patients received first-line SRS with a median OS of 13 months (18). In a Japanese study of 41 patients that all received first-line SRS for SCLC brain metastases the median OS was 8.1 months, the 12 -month local failure was $14 \%$, the 12 -month distant failure was $44 \%$, and the 2-year neurologic mortality was only $13 \%$ (19). A report of patients treated at the University of Pittsburgh between 1991 and 2018 included 28 patients (of 90 total patients) that received first-line SRS. The median OS for first-line SRS patients in this study is estimated to approximately 9 months based on the presented KaplanMeier curve (20). Cordeiro et al. reviewed the outcomes for 41 patients treated with SRS for SCLC brain metastases; six of 41 patients received first-line SRS. The median OS for the whole cohort was 6 months and there were no statistically significant differences between patients that had prior PCI, WBRT, or upfront SRS (21). In a large International Radiosurgery Research Foundation (IRRF) analysis, Cifarelli et al. reviewed the outcomes of 293 patients with SCLC brain metastases treated with Gamma Knife (Elekta AB, Stockholm, Sweden) radiosurgery; 61 of 293 patients received first-line SRS with a median OS of 
Table 1 Retrospective studies of SRS for SCLC brain metastases

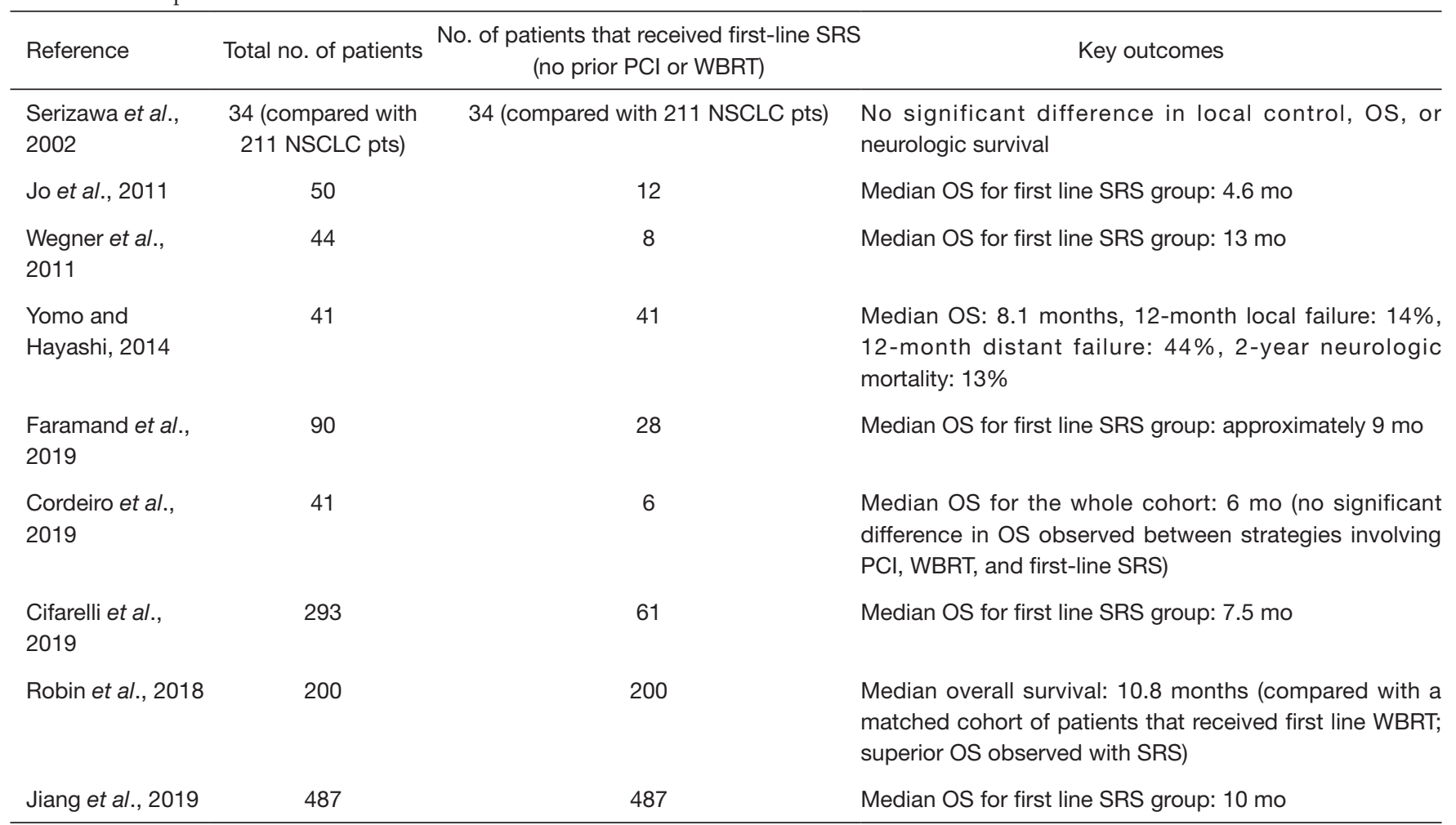

SRS, stereotactic radiosurgery; SCLC, small-cell lung cancer; NSCLC, non-small-cell lung cancer; PCI, prophylactic cranial irradiation; OS, overall survival; $\mathrm{PCI}$, prophylactic cranial irradiation; WBRT, whole brain radiation therapy; mo, months.

7.5 months (22). The radiation necrosis rate was reported at $5 \%$ among patients who received first-line SRS without prior PCI or WBRT.

Two national cancer registries studies have evaluated the outcomes after first-line SRS for SCLC brain metastases. Our group queried the national cancer database (NCDB) for patients with brain metastases at diagnosis and compared the outcomes after upfront SRS versus upfront WBRT with or without SRS. Upfront SRS was associated with superior OS on multivariate and propensity-score matched analyses. Although the survival advantage with SRS in this retrospective study may be related to patient selection and uncontrolled confounding, it does suggest that first-line SRS may be a reasonable option for some SCLC patients (23). Jiang et al. subsequently utilized the NCDB to present a patterns of care analysis of patients with SCLC that received CNS-directed radiation therapy. These investigators observed a slight increase in SRS utilization during the study period [2004-2013]. SRS use was associated with later study years, socioeconomic factors, treatment at an academic center, and residing in highereducated regions (24).

\section{The role of first-line SRS going forward}

Clarifying the role of first-line SRS in SCLC is likely to become more important going forward for several reasons. First, although PCI has historically been associated with improved survival $(2,3)$, these studies were largely performed in the pre-MRI era and utilized heterogenous staging and surveillance (7), whereas the OS benefit of PCI in the modern era of brain MRI surveillance and early salvage therapy was recently challenged by a Japanese phase 3 trial in extensivestage SCLC (8). As a result, the NCCN SCLC guidelines now recommend MRI surveillance for both limited and extensive-stage SCLC patients (regardless of PCI status) and the guidelines have changed PCI from a category-1 recommendation to an optional therapy for extensive stage SCLC (4). As a result of increased MRI surveillance and decreases in PCI administration, limited asymptomatic brain metastases will be identified more frequently in SCLC patients and more patients will be potential candidates for first-line SRS without prior brain radiation. Second, as immunotherapy becomes increasingly integrated into SCLC management following the positive results of phase 
3 trials in ES-SCLC $(5,6)$, improving prognoses in SCLC may increase the value of avoiding the long-term cognitive sequelae of radiation to the entire brain. Third, if advances in immunotherapy and other emerging systemic therapy agents can demonstrate enhanced CNS activity, as observed with other tumor histologies (25-27), systemic therapy could help to control occult microscopic CNS disease that was previously only achievable with PCI and WBRT.

\section{Ongoing studies}

While the small reports detailed above provide preliminary evidence in support of first-line SRS for SCLC brain metastases, importantly there are multiple ongoing studies in this area. Our group is leading the First-line Radiosurgery for Small-Cell Lung Cancer Brain Metastases (FIRE-SCLC) study through the IRRF to describe the outcomes with first-line SRS for SCLC brain metastases in a large international multicenter cohort (https:// www.irr-f.org/retrospective-trials-in-progress.html). The German ENCEPHALON study is a randomized phase II trial of WBRT versus SRS for 1-10 SCLC brain metastases (NCT03297788). A single arm phase II study being conducted at the Dana Farber Cancer Institute is studying SRS for patients with 1-6 SCLC brain metastases (NCT03391362). A recently activated phase 3 trial, SWOG S1827/MAVERICK (NCT04155034) is evaluating MRI surveillance with and without PCI in both limited and extensive stage SCLC. This trial is open across the National Clinical Trials Network (NCTN) cooperative groups including SWOG, Alliance, NRG, ECOG, and the CCTG, and will allow and track outcomes for salvage SRS and WBRT for brain metastases.

\section{Summary}

SRS has become the accepted standard for the management of CNS metastases arising from most tumor histologies, whereas WBRT has remained the standard of care for limited and even solitary SCLC brain metastases due to the exclusion of SCLC patients from landmark randomized trials. The primary concern with first-line SRS in SCLC has been the concern for short-interval progression and potential decrease in OS if WBRT is omitted. However, there is now a growing body of literature to suggest that not all patients with SCLC manifest rapid CNS progression and that some patients may be safely and effectively managed with first-line SRS, and this has led to the launch of several prospective trials of first line SRS in SCLC. In light of the forerunning literature of SRS with and without WBRT in other tumor histologies, it is reasonable to suspect that omitting upfront WBRT will be associated with a decrease in CNS control in SCLC as well. However, similar to other settings, clinicians and SCLC patients may ultimately be willing to accept a shorter time to new brain metastases if SRS can demonstrate less toxicity and comparable OS in prospective trials.

\section{Acknowledgments}

Funding: None.

\section{Footnote}

Provenance and Peer review: This article was commissioned by the Guest Editor (Jose M. Pacheco) for the series "Small Cell Lung Cancer", published in Fournal of Thoracic Disease. This article has undergone external peer review.

Conflicts of Interest: Both authors have completed the ICMJE uniform disclosure form (available at http://dx.doi. org/10.21037/jtd.2020.03.90). The series "Small Cell Lung Cancer" was commissioned by the editorial office without any funding or sponsorship. CGR reports that he is the national principle investigator of SWOG S1827/Maverick: MRI Brain Surveillance Alone versus MRI Surveillance and Prophylactic Cranial Irradiation (PCI): A Randomized Phase III Trial in Small-Cell Lung Cancer. The other author has no other conflicts of interest to declare.

Ethical Statement: The authors are accountable for all aspects of the work in ensuring that questions related to the accuracy or integrity of any part of the work are appropriately investigated and resolved.

Open Access Statement: This is an Open Access article distributed in accordance with the Creative Commons Attribution-NonCommercial-NoDerivs 4.0 International License (CC BY-NC-ND 4.0), which permits the noncommercial replication and distribution of the article with the strict proviso that no changes or edits are made and the original work is properly cited (including links to both the formal publication through the relevant DOI and the license). See: https://creativecommons.org/licenses/by-nc-nd/4.0/. 


\section{References}

1. Seute T, Leffers P, ten Velde GP, et al. Neurologic disorders in 432 consecutive patients with small cell lung carcinoma. Cancer 2004;100:801-6.

2. Auperin A, Arriagada R, Pignon JP, et al. Prophylactic cranial irradiation for patients with small-cell lung cancer in complete remission. Prophylactic Cranial Irradiation Overview Collaborative Group. N Engl J Med 1999;341:476-84.

3. Slotman B, Faivre-Finn C, Kramer G, et al. Prophylactic cranial irradiation in extensive small-cell lung cancer. $\mathrm{N}$ Engl J Med 2007;357:664-72.

4. NCCN.org. NCCN Clinical Practice Guidelines in Oncology (NCCN Guidelines), Small Cell Lung Cancer version 3.2020 [Online]. Available online: http://www. nccn.org. Accessed 03/01/2020.

5. Horn L, Mansfield AS, Szczesna A, et al. First-Line Atezolizumab plus Chemotherapy in Extensive-Stage Small-Cell Lung Cancer. N Engl J Med 2018;379:2220-9.

6. Paz-Ares L, Dvorkin M, Chen Y, et al. Durvalumab plus platinum-etoposide versus platinum-etoposide in firstline treatment of extensive-stage small-cell lung cancer (CASPIAN): a randomised, controlled, open-label, phase 3 trial. Lancet 2019;394:1929-39.

7. Rusthoven CG, Kavanagh BD. Prophylactic Cranial Irradiation (PCI) versus Active MRI Surveillance for Small Cell Lung Cancer: The Case for Equipoise. J Thorac Oncol 2017;12:1746-54.

8. Takahashi T, Yamanaka T, Seto T, et al. Prophylactic cranial irradiation versus observation in patients with extensive-disease small-cell lung cancer: a multicentre, randomised, open-label, phase 3 trial. Lancet Oncol 2017;18:663-71.

9. Rusthoven CG. Small Cell Lung Cancer: PCI Uncertainty and Emerging Radiosurgery Interest. Int J Radiat Oncol Biol Phys 2019;103:1034-5.

10. Aoyama H, Shirato H, Tago M, et al. Stereotactic radiosurgery plus whole-brain radiation therapy vs stereotactic radiosurgery alone for treatment of brain metastases: a randomized controlled trial. JAMA 2006;295:2483-91.

11. Brown PD, Ballman KV, Cerhan JH, et al. Postoperative stereotactic radiosurgery compared with whole brain radiotherapy for resected metastatic brain disease (NCCTG N107C/CEC.3): a multicentre, randomised, controlled, phase 3 trial. Lancet Oncol 2017;18:1049-60.

12. Brown PD, Jaeckle K, Ballman KV, et al. Effect of
Radiosurgery Alone vs Radiosurgery With Whole Brain Radiation Therapy on Cognitive Function in Patients With 1 to 3 Brain Metastases: A Randomized Clinical Trial. JAMA 2016;316:401-9.

13. Chang EL, Wefel JS, Hess KR, et al. Neurocognition in patients with brain metastases treated with radiosurgery or radiosurgery plus whole-brain irradiation: a randomised controlled trial. Lancet Oncol 2009;10:1037-44.

14. Kocher M, Soffietti R, Abacioglu U, et al. Adjuvant wholebrain radiotherapy versus observation after radiosurgery or surgical resection of one to three cerebral metastases: results of the EORTC 22952-26001 study. J Clin Oncol 2011;29:134-41.

15. Soffietti R, Kocher M, Abacioglu UM, et al. A European Organisation for Research and Treatment of Cancer phase III trial of adjuvant whole-brain radiotherapy versus observation in patients with one to three brain metastases from solid tumors after surgical resection or radiosurgery: quality-of-life results. J Clin Oncol 2013;31:65-72.

16. NCCN.org. NCCN Clinical Practice Guidelines in Oncology (NCCN Guidelines), Central Nervous System Cancers version 3.2020 [Online]. Available online: http:// www.nccn.org

17. Serizawa T, Ono J, Iichi T, et al. Gamma knife radiosurgery for metastatic brain tumors from lung cancer: a comparison between small cell and non-small cell carcinoma. J Neurosurg 2002;97:484-8.

18. Wegner RE, Olson AC, Kondziolka D, et al. Stereotactic radiosurgery for patients with brain metastases from small cell lung cancer. Int J Radiat Oncol Biol Phys 2011;81:e21-7.

19. Yomo S, Hayashi M. Upfront stereotactic radiosurgery in patients with brain metastases from small cell lung cancer: retrospective analysis of 41 patients. Radiat Oncol 2014;9:152.

20. Faramand A, Niranjan A, Kano H, et al. Primary or salvage stereotactic radiosurgery for brain metastatic small cell lung cancer. J Neurooncol 2019;144:217-25.

21. Cordeiro D, Xu Z, Shepard M, et al. Gamma Knife radiosurgery for brain metastases from small-cell lung cancer: Institutional experience over more than a decade and review of the literature. J Radiosurg SBRT 2019;6:35-43.

22. Cifarelli CP, Vargo JA, Fang W, et al. Role of Gamma Knife Radiosurgery in Small Cell Lung Cancer: A MultiInstitutional Retrospective Study of the International Radiosurgery Research Foundation (IRRF). Neurosurgery 2020;87:664-71.

23. Robin TP, Jones BL, Amini A, et al. Radiosurgery alone 
is associated with favorable outcomes for brain metastases from small-cell lung cancer. Lung Cancer 2018;120:88-90.

24. Jiang W, Haque W, Verma V, et al. Stereotactic radiosurgery for brain metastases from newly diagnosed small cell lung cancer: practice patterns and outcomes. Acta Oncol 2019;58:491-8.

25. Goldberg SB, Gettinger SN, Mahajan A, et al. Pembrolizumab for patients with melanoma or non-smallcell lung cancer and untreated brain metastases: early

Cite this article as: Robin TP, Rusthoven CG. Radiosurgery for small-cell lung cancer brain metastases: a review. J Thorac Dis 2020;12(10):6234-6239. doi: 10.21037/jtd.2020.03.90 analysis of a non-randomised, open-label, phase 2 trial. Lancet Oncol 2016;17:976-83.

26. Long GV, Atkinson V, Lo S, et al. Combination nivolumab and ipilimumab or nivolumab alone in melanoma brain metastases: a multicentre randomised phase 2 study. Lancet Oncol 2018;19:672-81.

27. Margolin K, Ernstoff MS, Hamid O, et al. Ipilimumab in patients with melanoma and brain metastases: an openlabel, phase 2 trial. Lancet Oncol 2012;13:459-65. 